\title{
Éclosion de cas de Salmonella Reading chez des personnes d'origine méditerranéenne orientale au Canada, 2014-2015
}

\author{
F Tanguay $^{1 \star}$, L Vrbova ${ }^{2}$, M Anderson 3 , Y Whitfield ${ }^{4}$, L Macdonald ${ }^{4,5}$, L Tschetter $^{6}$, A Hexemer $^{2}$ pour \\ l'équipe chargée de l'enquête sur l'éclosion de Salmonella Reading ${ }^{7}$
}

\section{Résumé}

Contexte : Salmonella Reading (S. Reading) est un sérotype rare au Canada de la sous-espèce (spp.) Salmonella, avec moins de neuf cas signalés chaque année (2011-2013). Au début 2015, une augmentation du nombre de cas de $S$. Reading a été repérée dans plusieurs provinces canadiennes, ce qui a mené au lancement d'une enquête nationale sur l'éclosion.

Objectif : Décrire une éclosion multiprovinciale de S. Reading au Canada qui a touché plus de 30 personnes.

Méthodologie : Les cas ont été définis par la confirmation en laboratoire de $S$. Reading présentant un profil d'électrophorèse en champ pulsé (PFGE) connexe. Les dates de début des symptômes s'étendaient de novembre 2014 à septembre 2015.

Au début de l'enquête, les enquêteurs ont remarqué que les cas étaient principalement d'origine méditerranéenne orientale, essentiellement afghane et libanaise, et que de nombreuses personnes touchées avaient consommé des aliments qui n'étaient pas habituellement nommés sur les questionnaires exploratoires normalisés en vue d'émettre des hypothèses sur la source d'une éclosion de maladies entériques. Des questions ouvertes sur la consommation d'aliments durant une période de trois jours ont été posées à un échantillon de répondants bénévoles de la communauté afin de mieux comprendre les préférences alimentaires des populations ethnoculturelles touchées. Les résultats de ce sondage ont servi à élaborer un questionnaire ciblé pour de nouvelles entrevues avec les cas et les cas subséquents de l'éclosion. Les enquêteurs en santé publique ont obtenu des échantillons du domicile des cas et des établissements alimentaires pertinents. Les autorités de salubrité des aliments ont mené une enquête de retraçage des aliments soupçonnés et ont prélevé des échantillons d'aliments à des fins d'analyse de laboratoire.

Résultats : On a recensé 31 cas confirmés (Ontario $=23$, Alberta $=7$, Nouveau-Brunswick $=1$ ) et trois cas probables (Ontario $=2$, Alberta $=1$ ) de $S$. Reading dans le cadre de cette éclosion. L'âge médian était de 31 ans (intervalle : moins de 1 an à 85 ans), 53 \% (18/34) des cas étaient des femmes. Sept cas ont été hospitalisés. Aucun décès n'a été signalé. La plupart des cas étaient d'origine méditerranéenne orientale $(n=23)$ ou avaient déclaré avoir consommé des aliments méditerranéens orientaux $(n=3)$. Les principales origines ethniques signalées par les cas étaient des Afghans en Ontario $(n=12)$ et des Libanais en Alberta $(n=3)$. La similarité génétique des isolats cliniques a été confirmée sans équivoque par le séquençage du génome entier.

Trois boulangeries ethniques ont été repérées en tant qu'exposition commune des cas; cependant, le retraçage des aliments d'intérêt dans ces boulangeries n'a pas permis de cerner un fournisseur commun, et la source de la maladie n'a pas été identifiée. Au total, 227 échantillons alimentaires provenant d'établissements de vente au détail $(n=142)$, de restaurants $(n=13)$ et du domicile de cas $(n=72)$ ont été analysés; deux échantillons alimentaires, des graines kalonji et du tahini, ont produit des résultats positifs pour S. Ruiru et $S$. Meleagridis. Ces produits ont été rappelés du marché.

Conclusion : Malgré des enquêtes épidémiologiques, microbiologiques et de retraçage alimentaire approfondies, une source commune n'a pas pu être identifiée pour cette éclosion de S. Reading. Les défis comprenaient le manque de connaissances des aliments consommés chez les groupes ethnoculturels touchés, ainsi que l'absence de données de référence sur les expositions alimentaires attendues dans la population de l'éclosion. La participation de partenaires locaux a contribué à comprendre les préférences alimentaires dans les communautés touchées. Étant donné la diversité ethnique et culturelle du Canada, des méthodes d'enquête sur les éclosions et des questionnaires sur la consommation d'aliments fondés sur un savoir-faire culturel pourraient être utiles.

\section{Affiliations}

${ }^{1}$ Centre des maladies infectieuses d'origine alimentaire, environnementale et zoonotique, Agence de la santé publique du Canada, Ottawa (Ontario)

${ }^{2}$ Centre des maladies infectieuses d'origine alimentaire, environnementale et zoonotique, Agence de la santé publique du Canada, Guelph (Ontario)

${ }^{3}$ Centre pour l'infrastructure en santé publique, Agence de la santé publique du Canada, Ottawa (Ontario)

${ }^{4}$ Santé publique Ontario, Toronto (Ontario)

${ }^{5}$ Dalla Lana School of Public Health, Université de Toronto, Toronto (Ontario)

${ }^{6}$ Laboratoire national de microbiologie, Agence de la santé publique du Canada, Winnipeg (Manitoba)

${ }^{7}$ Voir les remerciements

*Correspondance : florence.

tanguay@phac-aspc.gc.ca
Citation proposée : Tanguay F, Vrbova L, Anderson M, Whitfield Y, Macdonald L, Tschetter L et al. Éclosion de cas de Salmonella Reading chez des personnes d'origine méditerranéenne orientale au Canada, 2014-2015. Relevé des maladies transmissibles au Canada. 2017;43(1):15-22. https://doi.org/10.14745/ccdr.v43i01a03f 


\section{Introduction}

La salmonellose est l'une des causes les plus fréquentes d'éclosions d'origine alimentaire et cause habituellement une diarrhée, une fièvre et des douleurs abdominales. L'espèce la plus courante de ces bactéries gram négatif est $S$. enterica, qui se divise elle-même en six sous-espèces et de nombreux sérotypes. S. Reading est un sérotype rare au Canada : en 2012, sept cas ont été déclarés au Programme national de surveillance des maladies entériques (PNSME), et en 2013, neuf cas ont été déclarés $(1,2)$. Le PNSME est un système de surveillance fondé sur les analyses de laboratoire qui analyse et produit des rapports hebdomadaires à propos des cas confirmés d'entéropathogènes au Canada. L'objectif du présent article est de décrire une éclosion multiprovinciale de $S$. Reading au Canada qui a touché plus de 30 personnes.

\section{Détection de l'éclosion}

Le 21 janvier 2015, le PNSME a détecté une augmentation des cas signalés d'infection à $S$. Reading en Alberta $(n=2)$ et en Colombie-Britannique $(n=2)$. Une semaine plus tard, le 27 janvier 2015, le PNSME a relevé une augmentation des cas d'infection à S. Reading en Ontario $(n=4)$. Le 4 février 2015, Santé publique Ontario a ouvert une enquête sur l'éclosion en Ontario. Comme d'autres cas ont commencé à apparaître en Alberta, un comité national de coordination d'enquête en cas d'éclosion a été formé, en vertu des Modalités canadiennes d'intervention lors de toxi-infection d'origine alimentaire (MITIOA) (3).

\section{Méthodologie}

\section{Recherche des cas}

Des cas ont été déclarés entre le 21 janvier et le 25 août 2015. Les définitions de cas utilisées dans le cadre de la présente enquête étaient les suivantes :

\section{Définitions des cas de l'éclosion de Salmonella Reading \\ Un résident du Canada ou un visiteur avec : \\ - confirmation en laboratoire de S. Reading ET \\ - combinaison du profil PFGE'1 ReadXAI.0011/ ReadBNI.0005 OU ReadXAI.0012/ReadBNI.0005 OU ReadXAI.0014/ReadBNI.0005 ET \\ - $\quad$ symptômes apparus le $1^{\text {er }}$ novembre 2014 ou après cette date \\ Un résident du Canada ou un visiteur avec : \\ - confirmation en laboratoire de S. Reading ET Cas - PFGE ${ }^{1}$ en attente OU lien épidémiologique ${ }^{2}$ avec probable l'enquête actuelle \\ - $\quad$ symptômes apparus le $1^{\text {er }}$ novembre 2014 ou après cette date}

Abréviation : PFGE, électrophorèse en champ pulsé

2 Personne d'origine méditerranéenne orientale ou qui signale une exposition à des aliments de type méditerranéen oriental dans une province où un cas a été confirmé

\section{Questionnaires et expositions}

Les enquêtes initiales de santé publique ont été réalisées par les bureaux de santé publique pour tous les cas de salmonellose, conformément aux pratiques de base. Dans la mesure du possible, des questionnaires sur les cas de l'éclosion ont été recueillis et analysés de façon centralisée par l'équipe de l'enquête. Jusqu'au 4 mai 2015, les cas disponibles ont été soumis à une deuxième entrevue menée au moyen d'un questionnaire exploratoire uniformisé. Les entrevues étaient axées sur les aliments recensés durant le suivi initial des cas et que l'on croyait être fréquemment consommés par les personnes d'origine méditerranéenne orientale (voir la définition ci-dessous). Parmi ces aliments figuraient les graines de sésame, le tahini, les pistaches et les graines noires (oignon, nigelle, kalonji). Des questions supplémentaires ont été élaborées afin d'établir un lien possible entre les cas de l'Alberta et de l'Ontario (p. ex. un visiteur de l'Ontario ou des aliments apportés directement de I'Ontario en Alberta).

En mai 2015, des épidémiologistes de terrain ont été déployés afin d'aider l'équipe d'enquête et de collaborer avec les bureaux de santé publique et les partenaires locaux pour développer un questionnaire détaillé à questions ouvertes au sujet de la consommation alimentaire sur une période de trois jours. Les participants provenaient d'échantillons de volontaires des populations touchées par l'éclosion en vue de repérer des aliments additionnels habituellement consommés. Des entrevues en personne ont été menées, à l'aide de méthodes qui visaient à respecter les différences culturelles et à adapter les services en vue de répondre aux besoins uniques de la culture identifiée $(4,5)$. En Alberta, un agent d'hygiène du milieu parlant couramment l'arabe a participé aux entrevues avec les cas et aux sondages à propos de la consommation alimentaire sur une période de trois jours, et les cas ont été interviewés à leurs domiciles. En Ontario, des volontaires des groupes touchés (répondants de la communauté) ont été interviewés dans des milieux communautaires (p. ex. centres communautaires). Les résultats du questionnaire à propos de la consommation alimentaire sur trois jours ont orienté l'élaboration d'un questionnaire ciblé.

L'origine ethnique des cas était auto-déclarée pendant l'entrevue ou estimée en fonction de l'exposition aux aliments déclarée. Aux fins de cette enquête, l'origine méditerranéenne orientale a été définie en vertu des critères de l'Organisation mondiale de la Santé, soit des personnes qui identifient leur origine ethnique en lien avec des pays de la région de la Méditerranée orientale, c'est-à-dire l'Afghanistan, le Royaume de Bahreïn, Djibouti, I'Égypte, I'Iran (République islamique d'Iran), I'Iraq, la Jordanie, le Koweït, le Liban, la Libye, le Maroc, l'Oman, le Pakistan, le Qatar, I'Arabie saoudite, la Somalie, le Soudan, la République arabe syrienne, la Tunisie, les Émirats arabes unis et le Yémen (6).

\section{Enquête en laboratoire}

Une électrophorèse en champ pulsé (PFGE) a été réalisée sur tous les isolats de $S$. Reading. Une demande a ensuite été présentée à PulseNet États-Unis et à PulseNet International afin de trouver des profils PFGE correspondants à cet agrégat. 
Un avis EPIS (système de renseignements sur les épidémies du Centre européen de prévention et de contrôle des maladies [ECDC]) a été utilisé pour savoir si des profils PFGE correspondant à ceux des isolats de l'éclosion canadienne avaient été déclarés à l'ECDC. On a procédé au séquençage du génome entier (SGE) des cas et sélectionné des isolats de référence. Un arbre phylogénétique construit par la méthode de vraisemblance maximale à l'aide du pipeline SNVPhyl mis au point par la section bioinformatique du Laboratoire national de microbiologie a servi à déterminer le niveau de rapprochement des isolats, en fonction des positions des variants à nucléotide simple. La phylogénique SNVPhyl a été établie à l'aide de 642 hqSNV (variants à nucléotide simple du génome-cœur de haute qualité) détectés dans $93 \%$ du génome de référence (génome de l'isolat 15-0793 assemblé par l'algorithme SPAdes).

\section{Enquête sur la salubrité des aliments}

Des établissements alimentaires d'intérêt (commerces de vente au détail et restaurants) ont été recensés à partir des entrevues menées auprès des cas. L'enquête sur la salubrité des aliments a été initialement axée sur les produits contenant des graines de sésame, des graines de kalonji et du tahini. Le bœuf halal, les épices et les pistaches ont également été visés par l'enquête.

En partenariat avec le personnel de l'Agence canadienne d'inspection des aliments ( $\mathrm{ACI} A)$, les bureaux de la santé publique en Alberta et en Ontario ont visité les domiciles des cas et les établissements alimentaires (restaurants et commerces de vente au détail) recensés dans le cadre des entrevues menées auprès des cas. Aux établissements alimentaires, on a mené un examen des pratiques de manipulation des aliments à l'aide d'une analyse des risques aux points critiques (HACCP). On a prélevé des échantillons dans des établissements alimentaires et aux domiciles de cas à des fins de dépistage par les laboratoires provinciaux de l'Alberta et de l'Ontario. Une liste de fournisseurs a été obtenue auprès des établissements alimentaires et les fournisseurs communs ont été recensés. En outre, on a recueilli des renseignements sur la distribution des produits de boulangerie des établissements alimentaires d'intérêt.

L'ACIA a recueilli des renseignements sur les fournisseurs et les distributeurs des graines de sésame, des graines d'oignon/ kalonji, du tahini, des pistaches, du poulet halal et du bœuf halal auprès des établissements alimentaires en Ontario et en Alberta signalés par les cas. De plus, I'ACIA a recueilli des renseignements sur le fournisseur de deux boulangeries ethniques signalées par des cas de l'Ontario et de l'Alberta.

\section{Résultats}

\section{Épidémiologie descriptive}

Cette enquête comprenait 31 cas confirmés (Ontario $=23$, Alberta $=7$, Nouveau-Brunswick $=1$ ) et 3 cas probables (Ontario $=2$, Alberta $=1$ ). Les dates de début des symptômes s'étendaient du 7 novembre 2014 au 24 janvier 2015 (voir la figure 1). L'âge médian était de 31 ans (intervalle : moins de 1 an à 85 ans), $53 \%(18 / 34)$ des cas étaient des femmes. Sept cas ont été hospitalisés. Aucun décès n'a été signalé.

La plupart des cas signalés étaient d'origine méditerranéenne orientale ( $n=23$ ) ou avaient déclaré avoir consommé des aliments méditerranéens orientaux $(n=3)$. Parmi les cas, les sous-groupes ethniques prédominants étaient afghans $(n=12)$ en Ontario et libanais ( $n=3$ ) en Alberta (figure 2). Le cas du Nouveau-Brunswick a affirmé qu'il s'était rendu en Ontario durant la période d'exposition.

\section{Résultats de laboratoire}

Les trois combinaisons de profils PFGE figurant dans l'éclosion (ReadXI.0011/ReadBNI.0005, ReadXAI.0012/ReadBNI.0005 et ReadXAI.0014/ReadBNI.0005) étaient très semblables, et à la suite du SGE, on a établi qu'elles étaient génétiquement

Figure 1 : Cas confirmés et probables de l'éclosion de Salmonella Reading, par semaine de début des symptômes et par province, au Canada, du $1^{\text {er }}$ novembre 2014 au 11 septembre $2015(n=34)$

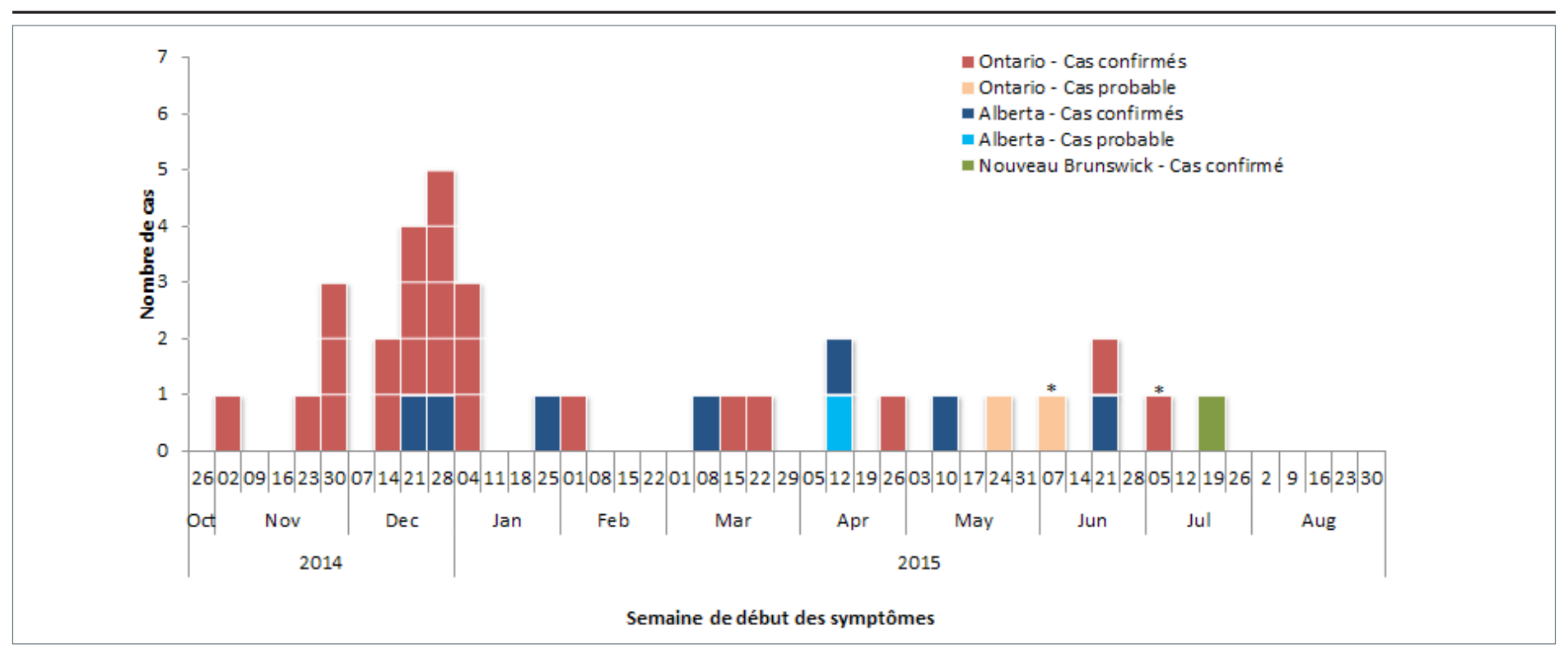

* Comme les cas étaient asymptomatiques, la date de prélèvement de l'échantillon a été utilisée au lieu de la date d'apparition des symptômes 
Figure 2 : Cas de Salmonella Reading liés à l'éclosion, par origine ethnique et par province, au Canada, du $1^{\text {er }}$ novembre 2014 au 11 septembre $2015(n=34)$

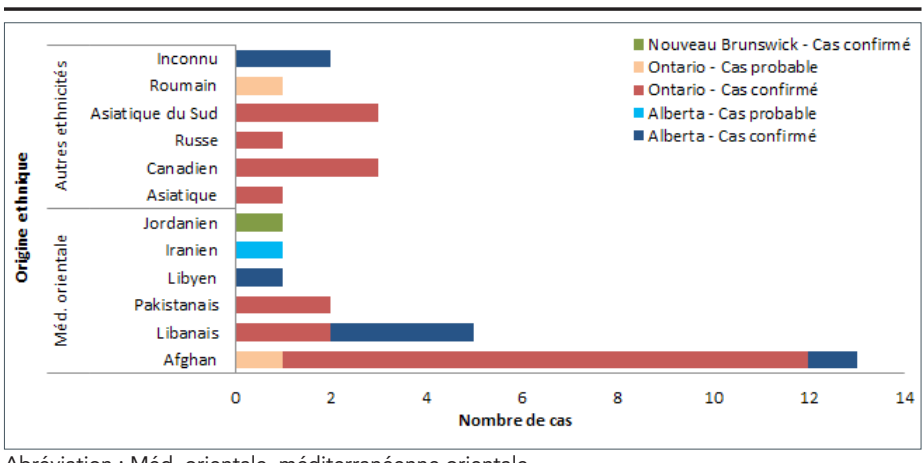

Abréviation : Méd. orientale, méditerranéenne orientale

identiques (figure 3). Aucune des trois combinaisons de profils PFGE n'avait déjà été décelée au Canada, aux États-Unis, dans les Caraïbes, en Amérique centrale ou en Amérique du Sud.

Figure 3 : Séquençage du génome entier (SGE) des cas de $S$. Reading, section de la génomique de la santé publique du Laboratoire national de microbiologie

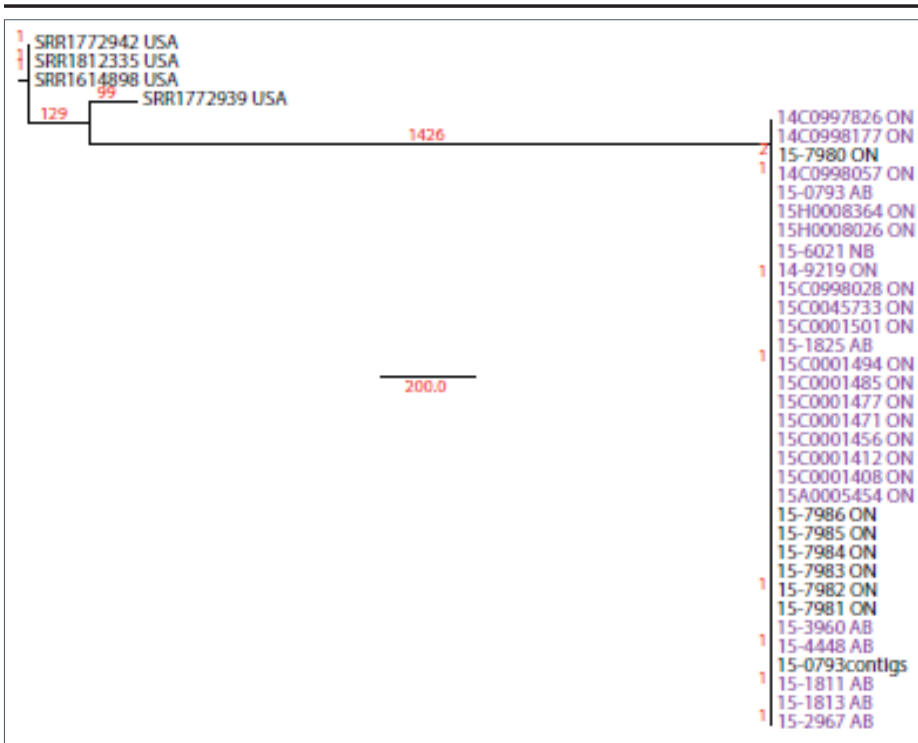

Référence : 15-0793. Méthode: Laboratoire national de microbiologie - pipeline SNVPhyl de la section bioinformatique, arbre phylogénétique construit par la méthode de vraisemblance maximale basée sur les positions de 642 variants à nucléotide simple du génome-cœur de haute qualité détectés dans les 37 isolats comparés à $93 \%$ du génome de référence. Contexte: Les isolats aux États Unis, 1502Read12MP (ReadXAI.0012, ReadBNI.005, ReadXAl.0011, ReadXAI.0014)

Les combinaisons de profils PFGE ReadXAI.0015/ ReadBNI.0007 $(n=1)$ et ReadXAI.0018/ ReadBNI.0010 $(n=2)$ sont de nouveaux profils qui ne sont pas étroitement liés aux profils associés aux cas confirmés; les cas présentant ces profils ont été inclus dans l'enquête comme cas probables, en raison de leur exposition à des aliments méditerranéens orientaux. Ces isolats n'ont pas été inclus dans le SGE.

\section{Antécédents d'exposition}

Les aliments signalés plus fréquemment par les cas étaient les suivants : le pain $(27 / 27,100 \%)$, la volaille et le poulet halal (23/26, $88 \%$ et $15 / 16,94 \%$, respectivement), le poivre noir
$(12 / 12,100 \%)$, le bœuf halal $(13 / 16,81 \%)$ et le pain pita (10/11, $91 \%)$. Le curcuma (10/13, $77 \%)$, les fruits séchés (13/19, $68 \%)$, les graines de sésame $(14 / 24,58 \%)$ et les pistaches $(12 / 21$, $57 \%$ ) ont également été signalés à une fréquence élevée, mais on n'a observé aucun point commun entre les cas.

Compte tenu de l'aspect de la courbe épidémique et du long intervalle d'apparition des cas, l'enquête était axée sur l'hypothèse voulant que des produits alimentaires de longue conservation soient la source probable de l'éclosion. Initialement, les graines de sésame, le tahini, les graines de kalonji/graines noires et les desserts en particuliers leurs ingrédients et garnitures, comme les pistaches, étaient des aliments soupçonnés d'être la source probable de l'éclosion. À la suite de nouvelles entrevues menées auprès des cas albertains et à partir des données recueillies au moyen des questions ouvertes sur les antécédents alimentaires menés auprès de membres de la communauté touchée, on a émis I'hypothèse que le bœuf halal puisse aussi être une source commune d'exposition.

Dans certaines situations, les cas ont hésité à fournir des renseignements sur les aliments consommés dans les trois jours précédant l'apparition de la maladie. Des entrevues menées avec des questions ouvertes auprès de répondants clés des communautés d'origine méditerranéenne orientale de l'Ontario et de l'Alberta ont révélé des thèmes récurrents laissant supposer l'existence d'obstacles potentiels à la communication de ces renseignements (voir l'encadré).

Observations qualitatives sur les antécédents d'exposition - obstacles potentiels à la collecte de renseignements à propos de l'exposition à des aliments sur une période de trois jours :

- La langue était perçue comme un obstacle courant, tant par les enquêteurs que par les répondants.

- Noms multiples pour le même aliment ou plat : incertitude ou manque de connaissances chez les enquêteurs, qui ont été atténuées par l'aide d'un interprète.

- Questions formulées avec des termes équivoques : incertitude chez certains répondants à propos des aliments ou des plats qui étaient considérés comme "typiques » ou " méditerranéens orientaux ».

- Manque de confiance perçue envers les agents du gouvernement chez certains nouveaux arrivants lorsqu'on leur posait des questions sur les aliments qu'ils avaient consommés récemment ou sur les pratiques en matière de cuisson, ou lorsqu'on leur demandait de remettre des échantillons d'aliments ou d'épices provenant de leurs cuisines.

- Sexospécificité : les rôles sexospécifiques de la préparation des aliments ont peut-être posé des défis dans la collecte de renseignements auprès d'hommes dans les foyers où les femmes s'occupent habituellement de la préparation des aliments.

- Biais lié aux réponses positives : certains répondants ont pu donner aux enquêteurs des réponses souhaitables sur le plan social aux questions sur la préparation des aliments qui ne reflétaient pas la pratique réelle, comme la cuisson entière de la viande, plutôt que la consommation de viande crue.

\section{Enquête sur la salubrité des aliments}

Les échantillons alimentaires pour les analyses en laboratoire ont été prélevés aux domiciles des cas, dans des restaurants et dans des établissements alimentaires de commerce de détail. Au total, plus de 200 échantillons ont été analysés par l'ACIA et les laboratoires provinciaux de l'Alberta et de l'Ontario, pour détecter la présence de Salmonella spp. Un résumé des échantillons prélevés et des résultats des analyses microbiologiques figure dans le tableau 1. On a détecté la présence de Salmonella Ruiru dans un échantillon de sauce de tahini (rappelée le 26 février 2014) et la présence de Salmonella 
Meleagridis dans un échantillon de graines kalonji entières (rappelées le 1er mars 2015).

Tableau 1 : Résumé des résultats d'analyse des échantillons alimentaires

\begin{tabular}{|c|c|c|c|}
\hline $\begin{array}{c}\text { Lieu } \\
\text { d'échantillonnage }\end{array}$ & $\begin{array}{l}\text { Aliment } \\
\text { échantillonné }\end{array}$ & $\begin{array}{c}\text { Nombre } \\
\text { d'échantillons }\end{array}$ & $\begin{array}{l}\text { Résultats des } \\
\text { analyses de } \\
\text { microbiologie } \\
\text { pour } \\
\text { Salmonella } \\
\text { spp. }\end{array}$ \\
\hline \multicolumn{4}{|c|}{ Analysés par le laboratoire provincial de l'Ontario } \\
\hline $\begin{array}{l}\text { Domiciles des cas } \\
\text { de l'Ontario }\end{array}$ & $\begin{array}{l}\text { Épices, graines } \\
\text { (sésame, } \\
\text { kalonji, etc.), } \\
\text { boulgour, } \\
\text { pistaches, fruits } \\
\text { séchés et autres } \\
\text { produits }\end{array}$ & 49 & Non détecté \\
\hline $\begin{array}{l}\text { Restaurants de } \\
\text { l'Ontario }\end{array}$ & $\begin{array}{l}\text { Ingrédients, } \\
\text { vinaigrettes } \\
\text { et différentes } \\
\text { graines utilisées } \\
\text { comme } \\
\text { garniture } \\
\text { pour la salade } \\
\text { fattouche }\end{array}$ & 13 & Non détecté \\
\hline $\begin{array}{l}\text { Commerces au } \\
\text { détail en Ontario }\end{array}$ & $\begin{array}{l}\text { Roti, biscuits, } \\
\text { diverses } \\
\text { graines, tahini, } \\
\text { épices et } \\
\text { autres produits } \\
\text { alimentaires de } \\
\text { vente au détail }\end{array}$ & 27 & Non détecté \\
\hline \multicolumn{4}{|c|}{ Analysés par le laboratoire provincial de l'Alberta } \\
\hline $\begin{array}{l}\text { Domiciles des cas } \\
\text { de l'Alberta }\end{array}$ & $\begin{array}{l}\text { Tahini, poitrines } \\
\text { de poulet, } \\
\text { kishke et } \\
\text { diverses épices }\end{array}$ & 21 & Non détecté \\
\hline $\begin{array}{l}\text { Commerces au } \\
\text { détail en Alberta }\end{array}$ & $\begin{array}{l}\text { Halva, } \\
\text { pistaches, bœuf } \\
\text { halal cru et } \\
\text { épices }\end{array}$ & 5 & Non détecté \\
\hline \multicolumn{4}{|c|}{ Analysés par l'Agence canadienne d'inspection des aliments } \\
\hline $\begin{array}{l}\text { Commerces au } \\
\text { détail en Ontario }\end{array}$ & $\begin{array}{l}\text { Graines } \\
\text { (sésame, } \\
\text { kalonji, etc.) } \\
\text { et produits de } \\
\text { tahini }\end{array}$ & 40 & $\begin{array}{l}\text { Non détecté } \\
\text { dans } \\
39 \text { échantillons } \\
\text { S. Meleagridis } \\
\text { détecté dans un } \\
\text { échantillon de } \\
\text { graines entières } \\
\text { de kalonji }\end{array}$ \\
\hline $\begin{array}{l}\text { Commerces au } \\
\text { détail en Alberta }\end{array}$ & $\begin{array}{l}\text { Graines } \\
\text { (sésame, } \\
\text { kalonji, etc.), } \\
\text { produits de } \\
\text { boulangerie } \\
\text { et de tahini, } \\
\text { épices et } \\
\text { pistaches }\end{array}$ & 63 & $\begin{array}{l}\text { Non détecté } \\
\text { dans } \\
62 \text { échantillons } \\
\text { S. Ruiru détecté } \\
\text { dans un } \\
\text { échantillon de } \\
\text { sauce de tahini }\end{array}$ \\
\hline
\end{tabular}

\section{Retraçage}

Aucune convergence n'a été relevée au sein des renseignements sur les fournisseurs et les distributeurs recueillis dans les établissements alimentaires de l'Ontario et de l'Alberta. À l'exception des principaux fournisseurs, aucun point commun n'a été observé entre les fournisseurs des boulangeries ethniques recensées par les cas en Ontario et en Alberta.

\section{Discussion}

Les éclosions de $S$. Reading ne sont pas courantes. Les éclosions de $S$. Reading déjà documentées avaient été associées à des germes $(7,8)$, à de la laitue iceberg (9), à du bœuf (10-13), à du porc (13), à de la dinde (14,15), à des huitres (16), à des chiens de berger (17) et à une source inconnue (18). Malgré des enquêtes épidémiologiques, microbiologiques et de retraçage approfondies, une source commune n'a pas pu être identifiée lors de cette enquête.

Cette enquête vient s'ajouter à la documentation explorant les facteurs culturels (y compris les obstacles) liés aux enquêtes et au contrôle des éclosions $(19,20)$. Elle illustre l'importance des compétences culturelles, c.-à-d. « [les] connaissances, habiletés et attitudes [nécessaires] pour fournir de l'information, des formations et des services dans le domaine de la santé à divers groupes (4) », pour une pratique efficace en santé publique et rappelle que les enquêtes sur les éclosions de maladies transmissibles sont facilitées par une approche adaptée aux différences culturelles $(21,22)$. Plusieurs stratégies ont permis de tenir compte de l'élément ethnoculturel essentiel à cette enquête. Le personnel de santé publique, notamment les épidémiologistes de terrain, a rencontré des obstacles lors de la génération d'hypothèses, en raison d'un manque de données de référence au sujet des préférences alimentaires des communautés ethnoculturelles touchées. Afin de surmonter cet obstacle, le questionnaire initial a été adapté pour comporter des aliments que l'on croyait être fréquemment consommés par les personnes d'origine méditerranéenne orientale. D'autres mesures ont ensuite été prises, sous la forme d'une enquête à propos des antécédents de consommation d'aliments sur une période de trois jours. Des entrevues ont été menées par un agent d'hygiène du milieu arabophone ou en partenariat avec un facilitateur connu du bureau de santé publique local, par le biais des réseaux établis avec la communauté. Les cas et des répondants de la communauté ont été interviewés à leur domicile ou dans un milieu communautaire familier afin d'établir la confiance et de favoriser la communication de renseignements. Les entrevues menées aux domiciles ont également donné l'occasion de prélever des échantillons d'aliments.

Bien qu'exigeantes sur le plan des ressources, ces méthodes se sont avérées inestimables afin d'atténuer les obstacles linguistiques et culturels et d'orienter l'élaboration du questionnaire sur l'éclosion. Fait intéressant, malgré les lieux géographiques disparates et les communautés ethnoculturelles différentes touchées par l'éclosion en Ontario et en Alberta, les enquêteurs des deux provinces ont choisi une méthode fondée sur le savoir-faire culturel qui s'est traduite par la collecte de renseignements similaires. Cela porte à croire que des méthodes semblables pourraient être adaptées en vue de répondre aux besoins de diverses communautés ethnoculturelles visées par une enquête sur une éclosion.

La participation des laboratoires provinciaux et de l'ACIA a joué un rôle déterminant dans cette enquête, étant donné le nombre d'échantillons alimentaires à analyser. Parmi les défis d'une enquête sur une éclosion prolongée, soulignons le fait que les 
échantillons du commerce au détail analysés tout au long de I'enquête sont probablement peu représentatifs du produit qui était offert au moment de l'exposition des cas. En outre, tous les cas ou les petits établissements de vente au détail n'ont pas été en mesure de fournir des données détaillant les dates et lieux exacts d'achat des aliments concernés, ce qui a limité la capacité de I'ACIA à effectuer des enquêtes de retraçage.

Enfin, l'importance du sous-typage moléculaire dans les enquêtes sur les éclosions ne saurait être sous-estimée. Comme dans de nombreux autres pays, les enquêtes sur les éclosions au Canada sont appuyées par des experts qualifiés chez PulseNet Canada. La capacité d'effectuer des analyses PFGE permet I'identification d'agrégats géographiquement disparates qui ne seraient autrement pas détectées. Reading est un sérotype rare de Salmonella, et il a fallu envoyer les isolats au Laboratoire national de microbiologie aux fins de confirmation ou de désignation du sérotypage. Ainsi, on a dû relever des défis, en ce qui a trait au signalement des cas en temps opportun. Par exemple, les longs délais entre le début des symptômes des cas et la confirmation qu'ils faisaient partie de l'éclosion se sont traduits par un retard dans les entrevues auprès des cas et l'échantillonnage alimentaire.

\section{Conclusion}

Malgré des enquêtes épidémiologiques, microbiologiques et de retraçage approfondies, une source commune pour cette éclosion de S. Reading n'a pas été identifiée. L'identification d'une source précise a été difficile en raison du manque de connaisance des enquêteurs concernant les aliments consommés par les personnes d'origine méditerranéenne orientale, d'obstacles linguistiques et culturels possibles lors des entrevues auprès des cas, ainsi que d'un manque de données de référence sur les expositions alimentaires attendues dans la population de l'éclosion.

Étant donné la diversité ethnique et culturelle du Canada, il pourrait être utile d'avoir recours à des méthodes d'enquête sur les éclosions et à des questionnaires sur la consommation d'aliments fondés sur un savoir-faire culturel. Plus particulièrement, l'inclusion systématique de questions à propos de l'origine ethnique ou des aliments ethniques sur les questionnaires exploratoires serait utile. Les données socio-démographiques (revenu, logement, origine ethnique, etc.) ne sont habituellement pas recueillies par les enquêteurs sur les éclosions de maladies transmissibles; cependant, lorsqu'ils sont pertinents aux données sur l'exposition, ces renseignements deviennent essentiels. On devrait également envisager d'ajuster les aliments sur lesquels ont pose des questions et d'adapter la terminologie afin de faciliter les entrevues auprès des communautés culturelles touchées. Des méthodes d'enquête additionnelles pourraient être indiquées, à la suite des entrevues initiales des cas, par exemple, des enquêtes sur la consommation d'aliments dans la population de la communauté touchée ou des entrevues en personnes fondées sur des questions ouvertes. II faut aussi combler les lacunes actuelles en matière de données de référence à l'échelle nationale sur la consommation alimentaire des groupes ethnoculturels minoritaires.

Il serait utile que l'équipe de l'enquête sur l'éclosion ou ses partenaires fassent preuve de compétences culturelles lors des éclosions qui présentent un élément ethnoculturel afin de repérer et de surmonter de potentiels obstacles. On pourrait pour ce faire envisager l'utilisation de données pertinentes provenant d'autres domaines de pratique de la santé publique (p. ex. promotion de la santé) ou établir un partenariat avec le bureau de santé publique local et ses réseaux communautaires existants afin de communiquer de manière efficace avec d'un groupe ethnoculturel ou linguistique particulier. Une formation appropriée en vue de développer le savoir-faire culturel chez les professionnels canadiens de la santé publique, particulièrement ceux qui participent aux enquêtes sur l'éclosion de maladies entériques, faciliterait la mise en œuvre des recommandations.

\section{Contributions des auteurs}

FT - Administration du projet, conceptualisation, méthodologie, enquête, rédaction (ébauche originale, puis révision et édition), LV - Administration du projet, conceptualisation, méthodologie, enquête, rédaction (ébauche originale, puis révision et édition), MA - Conceptualisation, méthodologie, enquête, rédaction (ébauche originale, puis révision et édition), YW - Enquête, rédaction (révision et édition), LM - Conceptualisation, Méthodologie, rédaction (révision et édition), LT - Ressources, enquête, rédaction, $\mathrm{AH}$ - Supervision, rédaction (revision et édition).

\section{Contributeurs}

\section{Équipe d'enquête sur l'éclosion}

Lance Honish, Organisme de santé publique Alberta Health Services - Enquête, rédaction (révision et édition)

Victor Mah, ministère de la Santé de l'Alberta - Enquête, rédaction (révision et édition)

Karen Johnson, Santé publique Ontario - Enquête

Stephen Moore, Santé publique Ontario - Enquête

Alison Samuel, Santé publique Ontario - Enquête

Aleisha Reimer, Laboratoire national de microbiologie Ressources

Chrystal Berry, Laboratoire national de microbiologie Ressources

Leah Isaac, Agence canadienne d'inspection des aliments Enquête

Sam Mohajer, Agence canadienne d'inspection des aliments Enquête

Pasha Marcynuk, Agence de la santé publique du Canada Méthodologie, enquête

Sujani Sivanantharajah, Agence de la santé publique du Canada Enquête

Melissa Phypers, Agence de la santé publique du Canada Supervision 


\section{Remerciements}

Les auteurs tiennent à remercier tous les membres du Comité national de coordination d'enquête en cas d'éclosion (CCEE) pour leurs conseils et leur soutien relatifs à cette enquête (collègues locaux de la santé publique de l'Ontario, de l'Alberta et du Nouveau-Brunswick; organisme de santé publique Alberta Health Services; ministère de la Santé de l'Alberta; Laboratoire provincial de santé publique de l'Alberta; ministère de la Santé du Nouveau-Brunswick; Santé publique Ontario; Laboratoire de santé publique de l'Ontario; ministère de la Santé et des Soins de longue durée de l'Ontario; ministère de l'Agriculture, de l'Alimentation et des Affaires rurales de l'Ontario; Agence canadienne d'inspection des aliments, Santé Canada et Agence de la santé publique du Canada).

En outre, les auteurs aimeraient également remercier les personnes suivantes pour leur contribution à cette enquête ou à la révision du manuscrit : Sarah Stephen, Samy Tawfik, Ingrid Zazulak, Amanda Yim et Dawn Greenwald de l'organisme de santé publique Alberta Health Services; Khalid Hussein du bureau de santé publique de Peel, Melissa Guy du bureau de santé publique d'Ottawa, Kathy Conlon du bureau de santé publique de Toronto et Margaret Mclntyre de Santé publique Ontario; Matthew Walker, Christy-Lynn Peterson, Alyssia Robinson, Cynthia Misfeldt et Celine Nadon du Laboratoire national de microbiologie de l'Agence de la santé publique du Canada.

\section{Conflit d'intérêts}

Aucun

\section{Financement}

Ce travail a été appuyé par l'Agence de la santé publique du Canada.

\section{Références}

1. Government of Canada. National Enteric Surveillance Program (NESP) Annual summary 2012. Guelph: Public Health Agency of Canada; 2014.

2. Government of Canada. National Enteric Surveillance Program (NESO) Annual summary 2013. Guelph: Public Health Agency of Canada; 2015.

3. Agence de la santé publique du Canada [Internet]. Modalités canadiennes d'intervention lors de toxi-infection d'origine alimentaire (MITIOA) 2010 en case d'éclosion multijuridictionnelle. Ottawa: ASPC; 2012 [mis à jour le 6 mars 2013; consulté le 21 juil 2016]. Disponible à l'adresse : http://www.phac-aspc.gc.ca/zoono/fiorp-mitioa/index-fra. php.

4. Agence de la santé publique du Canada [Internet]. Questions et réponses: Pratiques d'inclusion dans la prévention des infections transmissibles sexuellement et par le sang chez les minorités. Ottawa: ASPC; 2015 [mis à jour le 24 fev 2015; consulté le 21 juil 2016]. Disponible à l'adresse : http://www. phac-aspc.gc.ca/std-mts/rp/ipp-pip/ipp-pip-fra.php\#a4.

5. Alberta Health Services. Enhancing cultural competency: A resource kit for health care professionals [Internet]. Edmonton AB: AHS; 2005 [cited 2016 Jul 21]. Available from: http://fcrc.albertahealthservices.ca/pdfs/Enhancing_Cultural_ Competency_Resource_Kit.pdf.

6. Organisation mondiale de la Santé [Internet]. Bureau régional de la Méditerranée orientale. Genève: OMS; 2016 [consulté le 21 juil 2016]. Disponible à l'adresse : http://www. who.int/about/regions/emro/fr/.

7. Centers for Disease Control and Prevention [Internet]. Multistate outbreak of Salmonella Reading and Salmonella Abony infections linked to alfalfa sprouts (Final update). Atlanta GA: CDC; 2016 [mis à jour le 30 sept 2016; consulté 2016 Oct 18]. Disponible à l'adresse : http://www.cdc.gov/ salmonella/reading-08-16/.

8. Robertson LJ, Johannessen GS, Gjerde BK, Loncarevic S. Microbiological analysis of seed sprouts in Norway. Int J Food Microbiol. 2002 May;75(1-2):119-26.

9. Lienemann T, Niskanen T, Guedes S, Siitonen A, Kuusi M, Rimhanen-Finne R. Iceberg lettuce as suggested source of a nationwide outbreak caused by two Salmonella serotypes, Newport and Reading, in Finland in 2008. J Food Prot. 2011 Jun;74(6):1035-40.

10. Motsoela C, Collison EK, Gashe BA. Prevalence of Salmonella in two Botswana abattoir environments. J Food Prot. 2002 Dec;65(12):1869-72.

11. Gragg SE, Loneragan GH, Nightingale KK, Brichta-Harhay DM, Ruiz H, Elder JR, et al. Substantial within-animal diversity of Salmonella isolates from lymph nodes, feces and hides of cattle at slaughter. Appl Environ Microbiol. 2013 Aug;79(15):4744-50.

12. Corrier DE, Purdy CW, DeLoach JR. Effects of marketing stress on fecal excretion of Salmonella spp in feeder calves. Am J Vet Res. 1990 Jun;51(6):866-9.

13. Duggan S, Jordan E, Gutierrez M, Barrett G, O'Brien T, Hand $D$, et al. Salmonella in meats, water, fruit and vegetables as disclosed from testing undertaken by food business operators in Ireland from 2005 to 2009. Ir Vet J. 2012 Sep 22;65(1):17.

14. Centers for Disease Control (CDC). Foodborne nosocomial outbreak of Salmonella reading--Connecticut. MMWR Morb Mortal Wkly Rep. 1991 Nov 22;40(46):804-6.

15. Anderson PN, Hume ME, Byrd JA, Hernandez C, Stevens SM, Stringfellow K, et al. Molecular analysis of Salmonella serotypes at different stages of commercial turkey processing. Poult Sci. 2010 Sep;89(9):2030-7.

16. Oregon Health Authority/Public Health Division. Outbreaks: Foodborne and gastrointestinal - 2013. [Internet]. [consulté le 11 juin 2015]. Disponible à l'adresse : http://public.health. oregon.gov/DiseasesConditions/CommunicableDisease/ CDSummaryNewsletter/Documents/2014/ohd6319.pdf.

17. Salehi TZ, Badouei MA, Madadgar O, Ghiasi SR, Tamai IA. Shepherd dogs as a common source for Salmonella enterica 
serovar Reading in Garmsar, Iran. Turk J Vet Anim Sci. 2013;37(1):102-5.

18. Drachman RH, Petersen NJ, Boring JR, Payne FJ. Widespread Salmonella Reading infection of undetermined origin. Public Health Rep. 1958 Oct;73(10):885-94.

19. Culbertson NT, Scholl BJ. Cultural competency and patient-centered communication: A study of an isolated outbreak of urinary tract infections in Afghanistan. J Spec Oper Med. 2013. Fall;13(3):70-3

20. Charania NA, Tsuji LJ. A community-based participatory approach and engagement process creates culturally appropriate and community informed pandemic plans after the 2009 H1N1 influenza pandemic: Remote and isolated First Nations communities of sub-arctic Ontario, Canada. BMC Public Health. 2012 Apr 3;12:268.
21. Centers for Disease Control and Prevention. Outbreak of listeriosis associated with homemade Mexican-style cheese-North Carolina, October 2000-January 2001. MMWR Morb Mortal Wkly Rep. 2001;50(26):560-2.

22. Centers for Disease Control and Prevention. Outbreak of multidrug-resistant Salmonella enterica serotype Newport infections associated with consumption of unpasteurized Mexican-style aged cheese--Illinois, March 2006-April 2007. MMWR Morb Mortal Wkly Rep. 2008;57(16):432-5. 